\title{
Roles of Wnt/ $\beta$-Catenin Signaling in Controlling the Dopaminergic Neuronal Cell Commitment of Midbrain and Therapeutic Application for Parkinson's Disease
}

\author{
Liang-Wei Chen \\ Additional information is available at the end of the chapter \\ http://dx.doi.org/10.5772/53282
}

\section{Introduction}

Parkinson's disease (PD) is a severe deliberating neurodegenerative disease resulting from progressive and massive cell death of dopaminergic (DA) neurons in the substantia nigra [1]. While cell therapy strategy is strongly suggested for clinical cure of PD and DA progenitors are identified at different developing stages of midbrain, full understanding of key cell signaling or mechanism in controlling of DA neuronal differentiation from neural stem cells in vivo raises a great interest in cell therapeutic application for PD [2, 3]. A growing line of studies has shown that Wnts are lipid-modified factor for stem cell growth and cell differentiation and regulate midbrain DA neuronal development and hippocampal neurogenesis through both canonical and non-canonical Wnt signaling pathways [4-7]. The canonical Wnt $/ \beta$-catenin signaling appeals a key mechanism in controlling DA neuronal fate decision from neural stem cells or progenitors in the ventral midbrain during embryonic development and adulthood [8]. This chapter has focused on fast growing knowledge on $\mathrm{Wnt} / \beta$-catenin signaling pathway, intracellular cascade or crosstalk, functional roles in controlling DA neuronal fate decision and neural repair under physiological development and pathological events, and potential manipulation of Wnt/ $\beta$-catenin signaling pathway as cell therapeutic target for treatment of PD in human beings.

\section{The Wnt/ $\beta$-catenin signaling pathway}

The Wnt signaling pathway, conserved from low animals to primates, was originally identified as the morphogenic signaling for organogenesis. Among three branches of Wnt signal- 
ing pathways, the best studied one is the canonical Wnt pathway, which is highlighted by $\beta$ catenin-dependent regulation of down-streaming genes. The canonical Wnt ligands, e.g. Wnt1, Wnt2 and Wnt3a, can be secreted by surrounding neuronal and glial cells in the nervous system, bind to Frizzled and Lrp5/6 receptors in the target cells. At absence of binding with ligand Wnts, a protein complex in the cytoplasm, namely Axin2/GSK3 $\beta /$ APC complex makes phosphorylation of $\beta$-catenin and degradation of phosphorylated $\beta$-catenin, and keeps cytoplasmic $\beta$-catenin at such a low level that $\beta$-catenin can not be translocated into nucleus. Upon Wnt stimulation, the Axin2/GSK3 $3 /$ APC complex can be deaggregated, the cytoplasmic $\beta$-catenin is accumulated and increased $\beta$-catenin imported into nucleus. In the nucleus, $\beta$-catenin is recruited by transcription factors TCF1-4 to the promoter regions of the target genes for specific biological effects. These TCF-4 downstream targeting genes include c-myc, mmp-7, cyclin D1, CD44 that are actively and mainly involved in cell proliferation, cycling and cell differentiation (Figure 1). On the other hand, the non-canonical Wnt signaling pathways, i.e. PCP pathway and $\mathrm{Ca}^{2+}$ pathway, Wnt ligands bind to Frizzled receptors, then activate GTPase or increase intracellular Ca2+, transmitting signals by JNK cascade or without any nucleus events [4-6].

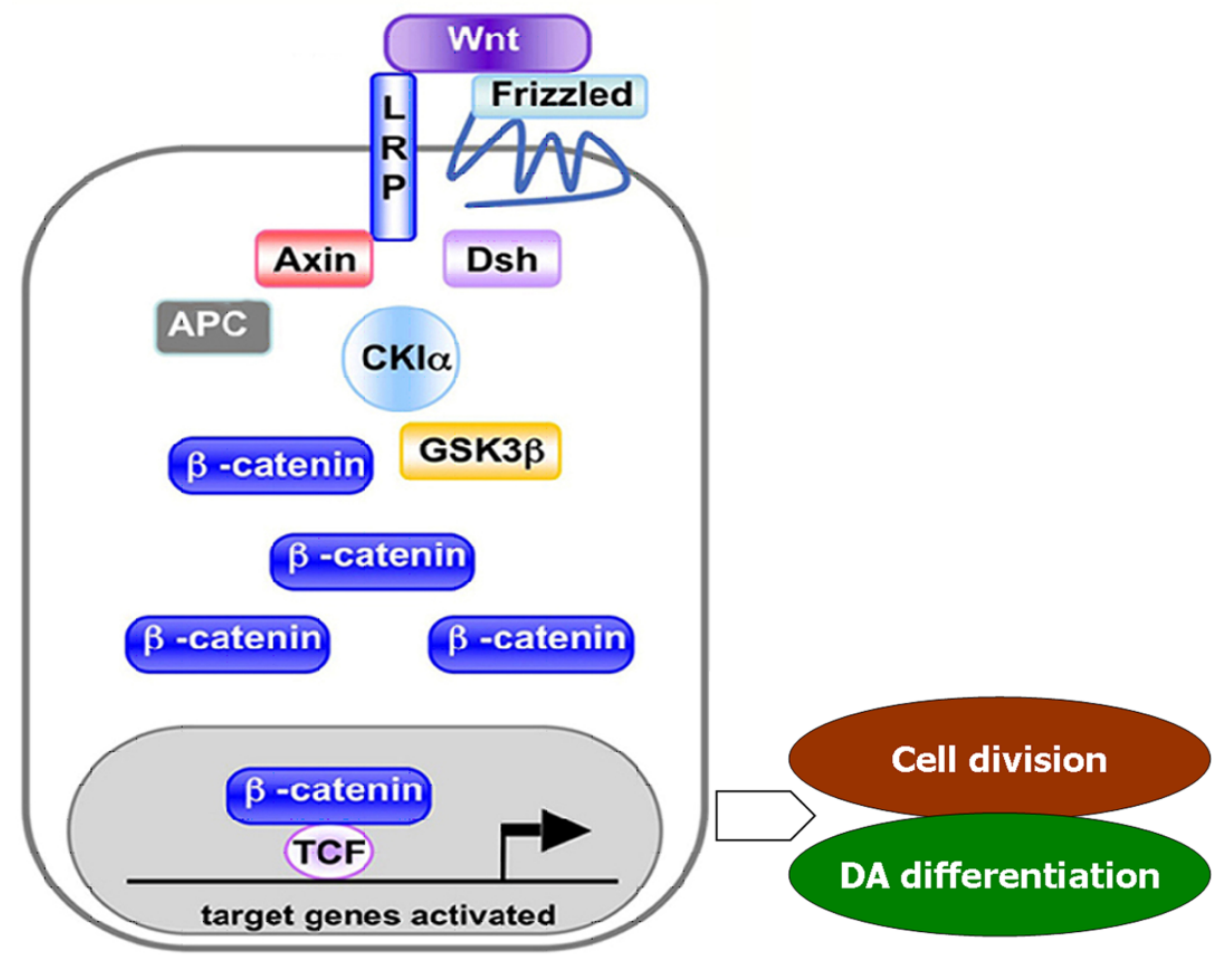

Figure 1. The canonical Wnt/ $\beta$-catenin signaling pathway in regulation of downstream target genes of DA neurogenesis (From Ref. 48, Ding, et al., 2011) 


\section{Role of $\mathrm{Wnt} / \beta$-catenin signaling in facilitating DA neuronal development}

The canonical Wnt $/ \beta$-catenin signaling pathways is critical for generation of DA neurons during development. Differential regulation of midbrain DA neurogenesis by Wnt1, Wnt3a, and Wnt5a was well studied [9]. The $\beta$-catenin was detected in DA precursor cells and $\beta$-catenin signaling took place in the precursor cells by assessment of TOPgal reporter mice. Wnt3a promoted proliferation of precursor cells expressing the orphan nuclear receptor-related factor 1 (Nurr1) but did not increase the number of DA neurons. The Wnt 1 and Wnt5a increased the number of midbrain DA neurons in E14.5 possibly by two mechanisms. Wnt1 predominantly increased the proliferation of Nurr1+ precursors that acquired a neuronal DA phenotype, up-regulated cyclins D1 and D3, and down-regulated p27 and p57 expression. In contrast, Wnt5a increased proportion of Nurr1+ precursors and up-regulated expression of Ptx3 and c-ret mRNA. Moreover, the soluble cysteine-rich domain of Frizzled-8 (a Wnt inhibitor) blocked endogenous Wnts and effects of Wnt1 and Wnt5a on proliferation and acquisition of DA phenotype. For the embryonic expression, Wnt1 was throughout of midbrain at E8.5, and then restricted to the roof plate, a subset of floor plate cells and isthemus of midbrain at E9.5. From E10 to E12, Wnt2 was observed in the ventral midbrain, with highest in the intermediate and marginal zone of ventral midbrain. Wnt3a was expressed in dorsal midbrain of rat atE11.5. The Wnt5a appeared at E9.5 and became restricted to the floor plate of midbrain from E11.5 to E13.5. Functionally, mutation of Wnt1 led to reduced DA neurons in late embryos. Mechanistic study showed that Wnt1 and its downstream gene Lmx1 formed a loop to regulate the expression of Octx2, Nurr1 and Pitx3, thereby establishing identity of DA precursors in vivo [10]. This Wnt1-lmx1a regulatory loop synergistically controlled DA differentiation in the midbrain by antagonizing Shh signaling pathway [11]. Wnt 2 mutation resulted in a decrease in proliferation of DA progenitors and subsequently loss of DA neurons, partially by phosphorylation of Lrp5/6 and Dishevelled 2/3. Wnt 3a promoted the proliferation of Nurr1-positive DA progenitors. The Wnt5a, derived by the astrocytes and radial glial cells, was demonstrated to promote cell fate commitment of precursors into DA neurons and development of A9-A10 DA neurons in vivo $[12,13]$. The Wnt5a also regulated DA axon growth and guidance in midbrain development [14]. In mouse embryo at E11.5, Wnt5a was abundantly expressed in the ventral midbrain where it promoted DA neurite and axonal growth. By E14.5, when DA axons were approaching their striatal target, Wnt5a caused DA neurite retraction. Co-culture of ventral midbrain explants with Wnt5a-overexpressing cell aggregates revealed that Wnt5a was capable of repelling DA neurites. Antagonism experiments revealed that the effects of Wnt5a were mediated by the Frizzled receptors and by small GTPase, Rac1. Moreover, this effect was specifically blocked by Wnt5a antibody. Role of Wnt5a in DA neuronal axon morphogenesis was further verified in Wnt5a-/-mice, where fasciculation of the medial forebrain bundle as well as the density of DA neurites and striatal terminals were disrupted. Although Wnts can function via intracellular $\beta$-catenin, Ca2+, and JNK signaling, the canonical $W n t / \beta$-catenin signaling pathway shows a major position in regulation of DA neurogenesis. It appeared that all Wnt1, Wnt3a and Wnt5a act in proliferation and DA differentiation of precursor cells with sequence of proliferation stimulating effect of $W n t 1 \geq W n t 3 a \geq W n t 5 a$, or differentiation facilitating effects of $W n t 5 a \geq W n t 1 \geq W n t 3 a$. These findings have evidenced that the $W n t / \beta-$ 
catenin signaling is a key regulator of proliferation and differentiation of DA precursors and different Wnts mighthave specific and unique activity profiles during DA neurogenesis.

Dynamic temporal and cell type-specific expression of Wnt signaling components was found in the developing midbrain [15]. The DA neuronal cluster size was determined during early forebrain patterning [16]. Furthermore, temporally controlling modulation of FGF/ERK signaling directed midbrain DA neural progenitor fate in mouse and human pluripotent stem cells [17]. Dickkopf-1 (Dkk1), a specific Wnt signaling inhibitor, regulated ventral midbrain DA neuronal differentiation and morphogenesis [18]. Blockade of Wnt/ $\beta$ catenin signaling pathway promoted neuronal induction and DA-phenotype differentiation in embryonic stem cells [19]. Delayed DA neuron differentiation was seen in the Lrp6 mutant mice [20]. Signaling interactions between $\mathrm{Wnt} / \beta$-catenin and sonic hedgehog (Shh) mechanisms functioned to regulate the production of DA neurons. Specific deletion of intracellular $\beta$-catenin in Shh expressing cells resulted in diminished DA progenitors, NgN2, BrdU labeling cells, and subsequently DA neurons. Permanent stabilization of $\beta$-catenin in Shh expressing cells led to more DA progenitors and DA neurons accordingly, and inhibition of GSK-3 $\beta$ also increases the differentiation of DA precursors [21]. HPRT deficiency coordinately dysregulated canonical Wnt signaling in neuro-developmental regulatory process [22]. Wnts showed antagonism of Shh signaling pathway and facilitated neurogenesis in the midbrain floor plate [23]. The $\beta$-arrestin was also a necessary component of Wnt/ beta-catenin signaling linking Dvl and axin in vitro and in vivo functions [24]. Wnt5a induced the DA differentiation of midbrain neural stem cells in vitro, and the effect was mediated by the phosphorylation of Dishevelled protein and activation of GTPase RAC1. Wnt5A stimulated the GDP/GTP exchange at pertussis toxin-sensitive heterotrimeric G proteins [25]. As to Wnt receptors, mutation of Lrp6 might not affect the patterning; proliferation and cell death in the ventral midbrain, but displayed a delay in the onset of DA precursor differentiation. Lrp6(-/-) mice exhibited 50\% reduction in DA neurons and expression of DA markers such as Nurr1 and Pitx3 as well as a defect in midbrain morphogenesis in the mutant embryos at E11.5. The extracellular domain of Lrp5/6 inhibited the non-canonical Wnt signaling in vivo condition [26]. The mitogen-activated protein kinases promoted $\mathrm{Wnt} / \beta$-catenin signaling via phosphorylation of LRP6 [27]. Ectopic Wnt/ $\beta$-catenin signaling was also involved in induction of neurogenesis in spinal cord [28]. In addition, Wnt5a was required for the endothelial differentiation of embryonic stem cells and vascularization via both Wnt/ $\beta$ catenin signaling and protein kinase $C$ pathways [29].

\section{Role of Wnt/ $\beta$-catenin signaling in DA neural plasticity or repair in adulthood}

The Wnt/ $\beta$-catenin signaling pathway also actively functions in neural plasticity and repair of DA neurons in the midbrain of disease conditions Interestingly, the crosstalk between Wnt $/ \beta$-catenin signaling and inflammatory was observed in plasticity of subventricular zone progenitors in response to 1-methyl-4-phenyl-1,2,3,6-tetrahydropyridine (MPTP) model of $\mathrm{PD}$, suggesting its involvement in consequences for neuroprotection and functional repair 
[30]. Accumulating evidence indicated that a population of astrocyte was functionally activated, named reactive astrocytes with active proliferation, morphological expanding of cell bodies and increasing generation of various neurotrophic factors, and with predominate distribution in the nigra and striatum of PD condition. These reactive astrocytes and $\mathrm{Wnt} / \beta$-catenin signaling showed a link of nigrostriatal injury to repair in MPTP model of PD. The Wnt signaling components Frizzled- 1 and $\beta$-catenin were dynamically regulated in response to MPTP insult-induced DA neuronal degeneration and reactive glial activation. Activated or reactive astrocytes in the ventral midbrain were identified as candidate source of Wnt1. Blocking Wnt/Fzd signaling with Dkk1 also counteracted astrocyte-induced neuroprotection against MPP (+) toxicity in primary mesencephalic astrocyte-neuron cultures. Moreover, astrocyte-derived Wnt1 promoted DA neurogenesis from adult midbrain stem cells or progenitor cells. Conversely, lack of Wnt1 transcription in response to MPTP in aged animals and failure of DA neurons to recover could be reversed by activation of $\mathrm{Wnt} / \beta$-catenin signaling in vivo, suggesting MPTP-reactive astrocytes and Wnt1 worked as neuroprotective activity in DA neural plasticity [31]. Obviously, Wnt1 regulated Frizzled-1/ $\beta$-catenin signaling pathway as a candidate regulatory circuit for DA neuron-astrocyte crosstalk in the ventral midbrain, also implying that Wnt signals might act as the critical messages in neuron-glial intercommunication in the adult mammalian nervous system [32].

Moreover, The Wnt/ $\beta$-catenin signaling also involved in Parkin protection of DA neurons [33]. Differential expression of Wnts was observed after spinal cord contusion injury in adult rodents [34]. Wnt signaling in the activated microglia; cells exhibited proinflammatory effect. Gene-expression profiling revealed that Wnt3A specifically increased expression of proinflammatory immune response genes in microglia and exacerbated release of IL-6, IL-12, and tumor necrosis factor $\alpha$ [35]. Heterotrimeric G protein-dependent Wnt5A signaling to ERK1/2 mediated distinct aspects of proinflammatory transformation in microglial cells [36]. While combining nitric oxide release with anti-inflammatory activity preserved DA innervation and prevented motor impairment in the MPTP model of PD [37], switching the microglial harmful phenotype promoted lifelong restoration of DA neurons from inflammatory degeneration in the substantia nigra of aged mice [38]. In addition, activation or inhibition of $W n t / \beta$-catenin signaling could regulate neuronal and glial differentiation in neurospheres, respectively. Inhibition of Wnt signaling promoted gliogenesis from neural stem cells. Long-term activation of Wnt signaling pathway by Wnt-7a or GSK3 inhibitors promoted a moderate increase of neuronal differentiation and blocked gliogenesis. In contrast, Wnt pathway inhibition by Dkk1overexpression robustly increased gliogenesis [39]. Accumulating evidences suggested that the glial cells including reactive astrocytes and microglial cells might present as crucial turning points for the therapeutic strategy against PD [40,41]

\section{Prospect on manipulation of $\mathrm{Wnt} / \beta$-catenin signaling for regeneration medicine}

The studies have indicated that transplantation of Wnt primed neural stem cells might result in improvements of cellular and functional recovery in PD condition. The midbrain neural 
stem cells with Wnt5a-treatment showed beneficial effect for DA cell replacement therapy in parkinsonian animal model [42], and purified Wnt5a increased dishevelled phosphorylation and DA neuronal differentiation in the midbrain [43]. Both GSK-3 $\beta$ inhibition and $\beta$-catenin stabilization increased commitment into DA neurons of neural precursors in the ventral midbrain [44], and the application of GSK-3 $\beta$ inhibitor lithium also influenced DA differentiation potential of human NT2 cells [45]. Generation of DA phenotype in neural stem cells could be carried out by engineering Nurr1 and Wnt signals [46]. Wnts also showed regulating role on differentiation of noradrenergic neuronal precursors in locus coeruleus [47]. In addition, the functional involvement of $\mathrm{Wnt} / \beta$-catenin signaling pathway in link of reactive astrocytes and plasticity or repair of nigrostriatal system further suggests $W n t / \beta$-catenin signaling as neuroprotection therapeutic targets [30,31]. It is hopefully that DA differentiation of the neural stem cells and the nigrostriatal plasticity may be effectively and specifically enhanced or improved by manipulation targeting on Wnt/ $\beta$-catenin signaling pathway [48].

\section{Summary}

Although the DA neuronal cell commitment from neural stem cells or progenitor cells is highly complicated and precisely regulated process, the canonical $\mathrm{Wnt} / \beta$-catenin pathway shows a critical role in controlling differentiation of DA neurons in the ventral midbrain during development and plasticity or neural repair in adulthood. In addition, Wnt singling might also act via Ca2+, JNK signaling and function candidate messages in DA neuron-glial cross-talk as well. Taken together, it is hopefully expected that molecular target manipulation of Wnt/ $\beta$-catenin signaling cascades will benefit controlling of DA neurogenesis and establishing novel cell therapy for PD in human beings.

\section{Acknowledgements}

This work was supported by grants from the Basic Research Program of China (2011CB504103, 2012CB525002) and the National Natural Science Foundation of China $(30970862,81071609,81272346)$.

\section{Author details}

Liang-Wei Chen

Address all correspondence to: lwchen@fmmu.edu.cn

Institute of Neurosciences, The Fourth Military Medical University, Xi'an, China 


\section{References}

[1] Fernandez-Espejo E. Pathogenesis of Parkinson's disease: prospects of neuroprotective and restorative therapies. Mol. Neurobiol., 2004; 29:15-30.

[2] Lie DC, Song H, Colamarino SA, Ming GL, Gage FH. Neurogenesis in the adult brain: new strategies for central nervous system diseases. Annu. Rev. Pharmacol. Toxicol., 2004; 44:399-421.

[3] Jönsson ME, Ono Y, Björklund A, Thompson LH. Identification of transplantable dopamine neuron precursors at different stages of midbrain neurogenesis. Exp. Neurol., 2009; 219:341-354.

[4] Willert K, Brown JD, Danenberg E, Duncan AW, Weissman IL, Reya T, Yates JR $3^{\text {rd, }}$ Nusse R. Wnt proteins are lipid-modified and can act as stem cell growth factors. Nature, 2003, 423:448-452.

[5] Prakash N, Wurst W. A Wnt signal regulates stem cell fate and differentiation in vivo. Neurodegener. Dis., 2007; 4:333-338.

[6] Widelitz R. Wnt signaling through canonical and non-canonical pathways: recent progress. Growth Factors, 2005; 23:111-116.

[7] Lie DC, Colamarino SA, Song HJ, Désiré L, Mira H, Consiglio A, Lein ES, Jessberger S, Lansford H, Dearie AR, Gage FH. Wnt signalling regulates adult hippocampal neurogenesis. Nature, 2005; 437:1370-1375.

[8] Castelo-Branco G, Arenas E. Function of Wnts in dopaminergic neuron development. Neurodegener. Dis., 2006; 3:5-11.

[9] Castelo-Branco G, Wagner J, Rodriguez FJ, Kele J, Sousa K, Rawal N, Pasolli HA, Fuchs E, Kitajewski J, Arenas E. Differential regulation of midbrain dopaminergic neuron development by Wnt-1, Wnt-3a, and Wnt-5a. Proc. Natl. Acad. Sci. USA., 2003; 100:12747-12752.

[10] Prakash N, Brodski C, Naserke T, Puelles E, Gogoi R, Hall A, Panhuysen M, Echevarria D, Sussel L, Weisenhorn DM, Martinez S, Arenas E, Simeone A, Wurst W. A Wnt1-regulated genetic network controls the identity and fate of midbrain-dopaminergic progenitors in vivo. Development, 2006; 133:89-98.

[11] Chung S, Leung A, Han BS, Chang MY, Moon JI, Kim CH, Hong S, Pruszak J, Isacson O, Kim KS. Wnt1-lmx1a forms a novel autoregulatory loop and controls midbrain dopaminergic differentiation synergistically with the SHH-FoxA2 pathway. Cell Stem Cell, 2009; 5:646-658.

[12] Andersson ER, Prakash N, Cajanek L, Minina E, Bryja V, Bryjova L, Yamaguchi TP, Hall AC, Wurst W, Arenas E. Wnt5a regulates ventral midbrain morphogenesis and the development of A9-A10 dopaminergic cells in vivo. PLoS One, 2008; 3:e3517. 
[13] Castelo-Branco G, Sousa KM, Bryja V, Pinto L, Wagner J, Arenas E. Ventral midbrain glia express region-specific transcription factors and regulate dopaminergic neurogenesis through Wnt-5a secretion. Mol. Cell. Neurosci., 2006; 31:251-262.

[14] Blakely BD, Bye CR, Fernando CV, Horne MK, Macheda ML, Stacker SA, Arenas E, Parish CL. Wnt5a regulates midbrain dopaminergic axon growth and guidance. PLoS One, 2011; 6:e18373.

[15] Rawal N, Castelo-Branco G, Sousa KM, Kele J, Kobayashi K, Okano H, Arenas E. Dynamic temporal and cell type-specific expression of Wnt signaling components in the developing midbrain. Exp. Cell Res., 2006; 312:1626-1636.

[16] Russek-Blum N, Gutnick A, Nabel-Rosen H, Blechman J, Staudt N, Dorsky RI, Houart C, Levkowitz G. Dopaminergic neuronal cluster size is determined during early forebrain patterning. Development, 2008; 135:3401-3413.

[17] Jaeger I, Arber C, Risner-Janiczek JR, Kuechler J, Pritzsche D, Chen IC, Naveenan T, Ungless MA, Li M. Temporally controlled modulation of FGF/ERK signaling directs midbrain dopaminergic neural progenitor fate in mouse and human pluripotent stem cells. Development, 2011; 138:4363-4374.

[18] Ribeiro D, Ellwanger K, Glagow D, Theofilopoulos S, Corsini NS, Martin-Villalba A, Niehrs C, Arenas E. Dkk1 regulates ventral midbrain dopaminergic differentiation and morphogenesis. PLoS One, 2011; 6:e15786.

[19] Cajánek L, Ribeiro D, Liste I, Parish CL, Bryja V, Arenas E. Wnt/beta-catenin signaling blockade promotes neuronal induction and dopaminergic differentiation in embryonic stem cells. Stem Cells, 2009; 27:2917-2927.

[20] Castelo-Branco G, Andersson ER, Minina E, Sousa KM, Ribeiro D, Kokubu C, Imai K, Prakash N, Wurst W, Arenas E. Delayed dopaminergic neuron differentiation in Lrp6 mutant mice. Dev. Dyn., 2010; 239:211-221.

[21] Tang M, Villaescusa JC, Luo SX, Guitarte C, Lei S, Miyamoto Y, Taketo MM, Arenas E, Huang EJ. Interactions of Wnt/beta-catenin signaling and sonic hedgehog regulate the neurogenesis of ventral midbrain dopamine neurons. J. Neurosci., 2010; 30:9280-9291.

[22] Kang TH, Guibinga GH, Jinnah HA, Friedmann T. HPRT deficiency coordinately dysregulates canonical Wnt and presenilin-1 signaling: a neuro-developmental regulatory role for a housekeeping gene? PLoS One, 2011; 6:e16572.

[23] Joksimovic M, Yun BA, Kittappa R, Anderegg AM, Chang WW, Taketo MM, McKay RD, Awatramani RB. Wnt antagonism of Shh facilitates midbrain floor plate neurogenesis. Nat. Neurosci., 2009; 12:125-31.

[24] Bryja V, Gradl D, Schambony A, Arenas E, Schulte G. Beta-arrestin is a necessary component of Wnt/beta-catenin signaling in vitro and in vivo. Proc. Natl. Acad. Sci. USA., 2007; 104:6690-6695. 
[25] Kilander MB, Dijksterhuis JP, Ganji RS, Bryja V, Schulte G. WNT-5A stimulates the GDP/GTP exchange at pertussis toxin-sensitive heterotrimeric G proteins. Cell Signal, 2011; 23:550-554.

[26] Bryja V, Andersson ER, Schambony A, Esner M, Bryjová L, Biris KK, Hall AC, Kraft B, Cajanek L, Yamaguchi TP, Buckingham M, Arenas E. The extracellular domain of Lrp5/6 inhibits noncanonical Wnt signaling in vivo. Mol. Biol. Cell., 2009; 20:924-936.

[27] Červenka I, Wolf J, Mašek J, Krejci P, Wilcox WR, Kozubík A, Schulte G, Gutkind JS, Bryja V. Mitogen-activated protein kinases promote WNT/beta-catenin signaling via phosphorylation of LRP6. Mol. Cell. Biol., 2011; 31(1):179-189.

[28] Joksimovic M, Patel M, Taketo MM, Johnson R, Awatramani R. Ectopic Wnt/beta-catenin signaling induces neurogenesis in the spinal cord and hindbrain floor plate. PLoS One, 2012; 7:e30266.

[29] Yang DH, Yoon JY, Lee SH, Bryja V, Andersson ER, Arenas E, Kwon YG, Choi KY. Wnt5a is required for endothelial differentiation of embryonic stem cells and vascularization via pathways involving both Wnt/beta-catenin and protein kinase Calpha. Circ. Res., 2009; 104:372-379.

[30] L'Episcopo F, Tirolo C, Testa N, Caniglia S, Morale MC, Deleidi M, Serapide MF, Pluchino S, Marchetti B. Plasticity of subventricular zone neuroprogenitors in MPTP (1-methyl-4-phenyl- 1,2,3,6-tetrahydropyridine) mouse model of Parkinson's disease involves cross talk between inflammatory and Wnt/ $\beta$-catenin signaling pathways: functional consequences for neuroprotection and repair. J. Neurosci., 2012; 32:2062-2085.

[31] L'Episcopo F, Tirolo C, Testa N, Caniglia S, Morale MC, Cossetti C, D'Adamo P, Zardini E, Andreoni L, Ihekwaba AE, Serra PA, Franciotta D, Martino G, Pluchino S, Marchetti B. Reactive astrocytes and $\mathrm{Wnt} / \beta$-catenin signaling link nigrostriatal injury to repair in 1-methyl-4-phenyl- 1,2,3,6-tetrahydropyridine model of Parkinson's disease. Neurobiol. Dis., 2011; 41:508-527.

[32] L'episcopo F, Serapide MF, Tirolo C, Testa N, Caniglia S, Morale MC, Pluchino S, Marchetti B. A Wnt1 regulated Frizzled-1/ $\beta$-Catenin signaling pathway as a candidate regulatory circuit controlling mesencephalic dopaminergic neuron-astrocyte crosstalk: Therapeutical relevance for neuron survival and neuroprotection. Mol. Neurodegener., 2011; 6:49.

[33] Rawal N, Corti O, Sacchetti P, Ardilla-Osorio H, Sehat B, Brice A, Arenas E. Parkin protects dopaminergic neurons from excessive Wnt/beta-catenin signaling. Biochem. Biophys. Res. Commun., 2009; 388:473-478.

[34] Fernández-Martos CM, González-Fernández C, González P, Maqueda A, Arenas E, Rodríguez FJ. Differential expression of Wnts after spinal cord contusion injury in adult rats. PLoS One, 2011; 6:e27000. 
[35] Halleskog C, Mulder J, Dahlström J, Mackie K, Hortobágyi T, Tanila H, Kumar Puli L, Färber K, Harkany T, Schulte G. WNT signaling in activated microglia is proinflammatory. Glia, 2011; 59:119-131.

[36] Halleskog C, Dijksterhuis JP, Kilander MB, Becerril-Ortega J, Villaescusa CJ, Lindgren E, Arenas E, Schulte G. Heterotrimeric G protein-dependent WNT-5A signaling to ERK1/2 mediates distinct aspects of microglia proinflammatory transformation. J. Neuroinflammation., 2012; 9:111.

[37] L'Episcopo F, Tirolo C, Caniglia S, Testa N, Serra PA, Impagnatiello F, Morale MC, Marchetti B. Combining nitric oxide release with anti-inflammatory activity preserves nigrostriatal dopaminergic innervation and prevents motor impairment in a 1methyl-4-phenyl-1,2,3,6- tetrahydropyridine model of Parkinson's disease. J Neuroinflammation. 2010 Nov 23; 7:83.

[38] L'episcopo F, Tirolo C, Testa N, Caniglia S, Morale MC, Impagnatiello F, Marchetti B. Switching the microglial harmful phenotype promotes lifelong restoration of subtantia nigra dopaminergic neurons from inflammatory neurodegeneration in aged mice. Rejuvenation Res., 2011; 14:411-424.

[39] Kunke D, Bryja V, Mygland L, Arenas E, Krauss S. Inhibition of canonical Wnt signaling promotes gliogenesis in P0-NSCs. Biochem. Biophys. Res. Commun., 2009; 386:628-633.

[40] L' Episcopo F, Tirolo C, Testa N, Caniglia S, Morale MC, Marchetti B. Glia as a turning point in the therapeutic strategy of Parkinson's disease. CNS Neurol. Disord. Drug Targets, 2010; 9:349-372.

[41] Chen LW, Yung KL, Chan YS. Reactive astrocytes as potential manipulation targets in novel cell replacement therapy of Parkinson's disease. Current Drug Targets, 2005; 6:821-833.

[42] Parish CL, Castelo-Branco G, Rawal N, Tonnesen J, Sorensen AT, Salto C, Kokaia M, Lindvall $\mathrm{O}$, Arenas E. Wnt5a-treated midbrain neural stem cells improve dopamine cell replacement therapy in parkinsonian mice. J. Clin. Invest., 2008; 118:149-160.

[43] Schulte G, Bryja V, Rawal N, Castelo-Branco G, Sousa KM, Arenas E. Purified Wnt-5a increases differentiation of midbrain dopaminergic cells and dishevelled phosphorylation. J. Neurochem., 2005; 92:1550-1553.

[44] Castelo-Branco G, Rawal N, Arenas E. GSK-3beta inhibition/beta-catenin stabilization in ventral midbrain precursors increases differentiation into dopamine neurons. J. Cell Sci., 2004; 117:5731-5737.

[45] Misiuta IE, Saporta S, Sanberg PR, Zigova T, Willing AE. Influence of retinoic acid and lithium on proliferation and dopaminergic potential of human NT2 cells. J. Neurosci. Res., 2006; 83:668-679.

[46] Arenas E. Engineering a dopaminergic phenotype in stem/precursor cells: role of Nurr1, glia-derived signals, and Wnts. Ann. N. Y. Acad. Sci., 2005; 1049:51-66. 
[47] Holm PC, Rodríguez FJ, Kele J, Castelo-Branco G, Kitajewski J, Arenas E. BMPs, FGF8 and Wnts regulate the differentiation of locus coeruleus noradrenergic neuronal precursors. J. Neurochem., 2006; 99:343-352.

[48] Ding YX, Wei LC, Wang YZ, Cao R, Wang X, Chen LW. Molecular manipulation targeting regulation of dopaminergic differentiation and proliferation of neural stem cells or pluripotent stem cells. CNS Neurol. Disord. Drug Targets, 2011; 10:517-528. 
